\title{
Application of Beta Regression to Analyze Ischemic Stroke Volume in NINDS rt-PA Clinical Trials
}

\author{
Christopher J. Swearingen ${ }^{a}$ Barbara C. Tilley ${ }^{b}$ Robert J. Adams ${ }^{c}$ \\ Zoran Rumboldt $^{d}$ Joyce S. Nicholas ${ }^{e}$ Dipankar Bandyopadhyay ${ }^{f}$ \\ Robert F. Woolson ${ }^{\mathrm{e}}$ \\ a Pediatric Biostatistics Program, University of Arkansas for Medical Sciences, Little Rock, Ark., \\ ${ }^{b}$ Department of Biostatistics, University of Texas School of Public Health, Houston, Tex., Departments of ${ }^{\mathrm{c} N e u r o l o g y,}$ \\ ${ }^{\mathrm{d}}$ Radiology, and e Division of Biostatistics and Epidemiology, Medical University of South Carolina, Charleston, S.C., \\ fDivision of Biostatistics, University of Minnesota School of Public Health, Minneapolis, Minn., USA
}

\section{Key Words}

Beta regression $\cdot$ Clinical trial $\cdot \mathrm{NINDS} \cdot \mathrm{rt}-\mathrm{PA} \cdot$ Stroke

\begin{abstract}
Background and Purpose: Ischemic stroke lesion volumes have proven difficult to analyze due to the extremely skewed shape of their underlying distribution. We introduce an extension of generalized linear models, beta regression, as a possible method of modeling extremely skewed distributions as evidenced in ischemic stroke lesion volumes. Methods: The NINDS rt-PA clinical trials measured ischemic stroke lesion volume as a secondary trial outcome. Three-month lesion volumes from these trials were analyzed using beta regression. A multi-variable regression model associating explanatory variables with ischemic stroke lesion volumes was constructed using accepted model building strategies and compared with the previously published volumetric analysis. Results: Beta regression produced a similar model when compared to the previous analysis published by the study group. All previously identified variables of importance were detected in the model building process. The age by treatment interaction de-
\end{abstract}

scribed in previous studies was also found in this analysis, confirming the strong effect age has on stroke outcomes. Further, a treatment effect was elicited in terms of odds ratios, yielding a previously unknown quantification of the effect of rt-PA on lesion volumes. Conclusions: Beta regression proved adept in modeling ischemic stroke lesions and offered the interpretation of covariates in terms of odds ratios. Beta regression is seen as a legitimate alternative to analyze ischemic stroke volumes.

Copyright $\odot 2011$ S. Karger AG, Basel

\section{Introduction}

Advances in neuroimaging have led to improved stroke classification $[1,2]$ and more accurate measurement of the volume of an acute ischemic stroke lesion [2-4]. The change in lesion volume is used to demonstrate possible efficacy of a novel treatment in ischemic stroke animal models [5] and is believed to be associated with clinical outcomes and may potentially represent a prognostic indicator of ischemic stroke outcome [2, 3, 6-8]. While the diagnostic impact of advanced neuroimaging

\section{KARGER}

두 2011 S. Karger AG, Basel

Fax +4161306 1234 E-Mail karger@karger.ch www.karger.com
Christopher J. Swearingen, $\mathrm{PhD}$

Department of Pediatrics, University of Arkansas for Medical Sciences

Biostatistics, Slot 512-43, 1 Children's Way

Little Rock, AR 72202 (USA)

Tel. +1 501364 6639, E-Mail cswearingen@ uams.edu 
is touted, the clinical prognostic relevance of infarct volume is not as well understood [2-6].

A complication to the understanding of the clinical relevance of infarct volume is the statistical challenge of analyzing this measure. A review of clinical literature reporting at least one measure of central tendency (mean or median) and one measure of dispersion [standard deviation, range or interquartile range (IQR)] for infarct volumes identified 23 articles (online supplementary table; for all supplementary material, see www. karger.com/doi/10.1159/000330375). Most of these reported standard deviations are equal in magnitude or larger than the mean infarct volume, and most median infarct volumes are not located in the middle of the accompanying range. Additionally, several ranges include 0 cubic centimeters $\left(\mathrm{cm}^{3}\right)$, the minimum measurement boundary, indicating how the infarct volume distribution is 'truncated', having a mass of observations at the boundary.

Attention may be brought to differences between imaging modalities and study protocols as contributing to the skewed infarct volume distribution. Diffusionweighted magnetic resonance imaging (DW-MRI) can detect an ischemic lesion early after stroke $[6,9-12]$ and has been shown to be more sensitive to early ischemic changes than computed tomography (CT) scans $[5,6]$. However, regardless of imaging technology, the infarct distribution remains severely skewed, which is in part due to the fact that infarct volume is subject to the same strictly non-negative measurement boundaries of total brain volume. These boundary restrictions contribute to infarct volumes having an extremely non-normal distribution, leading to the consistent and repeated reporting of infarct volume distributions as 'resistant to all forms of transformation' [8] and 'ill-behaved ... [lacking any] underlying distribution' [13].

Recent statistical developments may provide a needed solution to the intractably skewed infarct volume distribution. In this paper, beta regression is introduced that presumes the variable of interest, infarct lesion volumes, can be described by a beta distribution. The beta distribution is extremely flexible and may possibly provide an underlying distribution for modeling infarct volumes. We utilize beta regression in modeling ischemic stroke volumes from the National Institute of Neurological Diseases and Stroke recombinant tissue type plasminogen activator (NINDS rt-PA) clinical trials, comparing the results of our analysis to previously published reports.

\section{Methods}

NINDS rt-PA Clinical Trials

The NINDS rt-PA trials were two concurrent randomized, placebo-controlled, double-blind clinical trials conducted between January 1991 and October 1994 assessing the effectiveness of rt-PA in treating cerebral artery thrombosis (clot restricting or stopping blood flow) given within $3 \mathrm{~h}$ of ischemic stroke onset in 624 participants [14]. The trials purposed to document the therapeutic efficacy of rt-PA as well as its safety as cerebral hemorrhage was a known complication in early ischemic stroke treatment trials [14]. Further, open-label studies had provided evidence of safe dose levels and target treatment windows, contributing to the scientific and clinical equipoise needed to further test rt-PA in large clinical trial settings [14].

The trials were similarly constructed with each using the same procedures and following the same protocol but differing on primary endpoints. The part 1 trial assessed 24-hour functional improvement as measured by the National Institutes of Health Stroke Scale (NIHSS) [15] as its primary endpoint; the part 2 trial assessed the combined functional outcomes at 3 months as measured by the NIHSS and other clinical outcomes [14]. Further detailed description of the NINDS rt-PA clinical trials are available [14].

The rt-PA trials measured infarct volume using CT at baseline, $24 \mathrm{~h}, 1$ week (within a day $7-10$ window), and 3 months for a preplanned secondary analysis assessing infarct volume between treatment groups. Baseline CT scans were obtained with a $10-\mathrm{mm}$ slice thickness, while all other time points were obtained with a 5-mm slice thickness. The lesion volume collected at each study time point was observed to have an extremely skewed distribution with several observations at $0 \mathrm{~cm}^{3}$ (fig. 1a, b).

The original analysis of the 'intention-to-treat' lesion volumes [16] at 3 months employed a cube-root transformation in a loglinked generalized estimating equation (GEE) model. Treatment group differences adjusting for other associated covariates were investigated, but, for reasons not clearly elucidated, 18 participants with a stroke subtype classification of 'other', as defined by the Trial of Org 10172 in Acute Stroke Treatment (TOAST) criteria [17], were excluded from the original NINDS analysis. This multi-variable GEE analysis was able to detect an age by rt-PA interaction effect on the 3-month lesion volumes, indicating that younger trial participants given rt-PA had smaller stroke volumes at 3 months, while older trial participants given rt-PA had larger stroke volumes when compared to placebo participants of the same age. However, the effect was not quantified as only general discussion of the age/rt-PA effect was given. Further variables determined by the NINDS rt-PA study group to be associated with 3 -month lesion volume were TOAST subtype and an interaction between early lesion findings on CT and NIHSS.

\section{Beta Regression}

Formally introduced in political science [18], beta regression differs from traditional linear regression as it models a dependent variable that follows a beta distribution, not a normal distribution. The beta distribution can be parameterized by its mean and variance similar to a normal distribution. However, unlike the normal distribution, the variance of a beta distribution is a function of its mean and a 'precision' parameter, a scaling measurement describing how tightly clustered the observed data are. Beta 


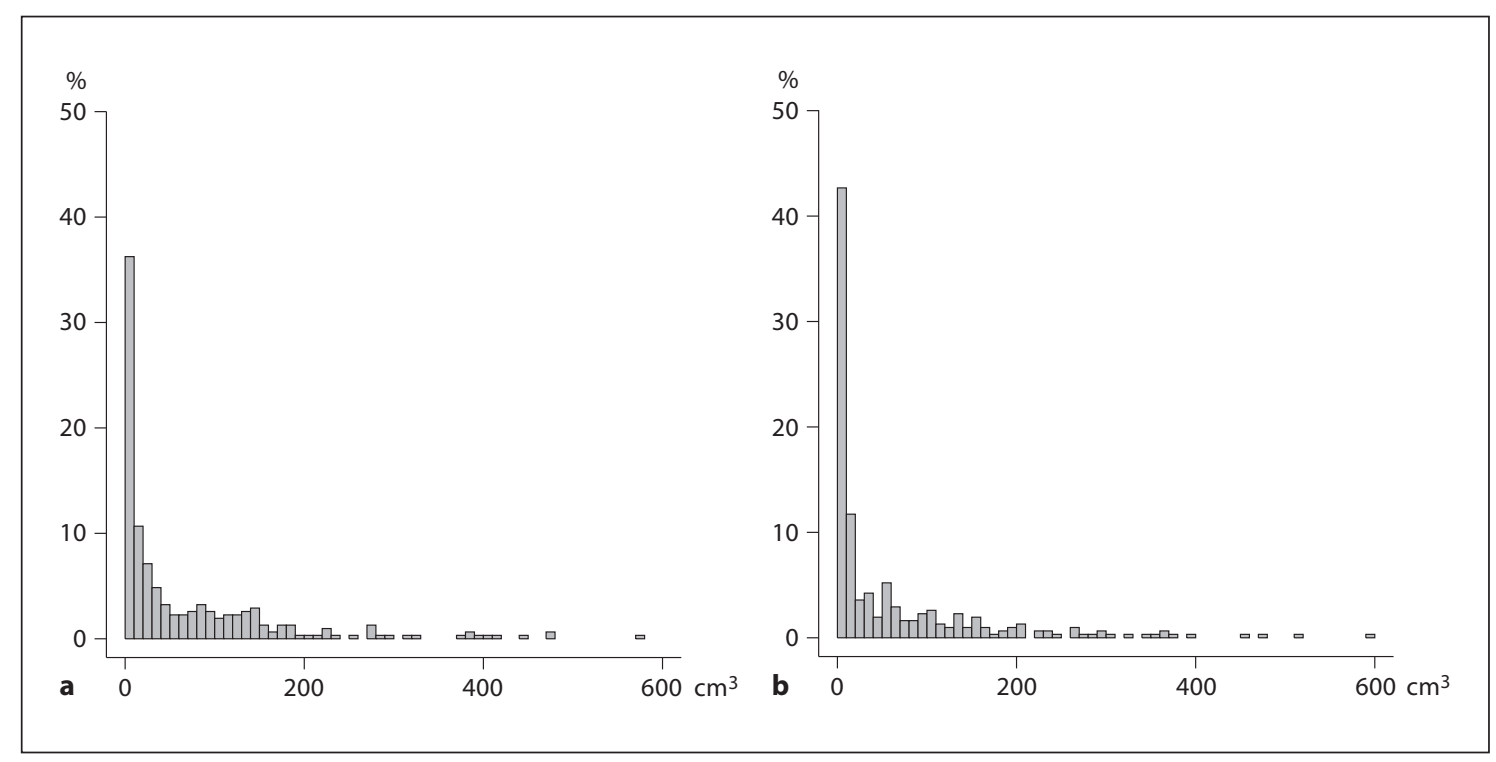

Fig. 1. Histograms of stroke lesion volume in the NINDS rt-PA clinical trials at 3 months by treatment group. a Placebo at 3 months. b rt-PA at 3 months.

regression uses this particular aspect of the beta distribution to model the association of explanatory variables to changes in mean and variance simultaneously.

Beta regression falls into the class of regression methods termed 'generalized linear model' (GLM). The basic setup of a GLM is mathematically defined as:

$$
Y=f\left(X_{1}, \ldots, X_{n}\right)
$$

where the function $f$ is used to relate any number $n$ of explanatory variables $X_{1}$ through $X_{n}$ to changes in a dependent variable $Y$. In linear regression, a specific case of a GLM, an untransformed link function (identity function) is used to model the dependent variable in terms of the explanatory variables in which a unit change in any $X$ explains a unit change in $Y$ accounting for error and other $X$ variables in the model. Beta regression differs from linear regression in this regard, as it uses two functions to link explanatory variables to the mean and precision parameters, respectively, of the dependent variable [18]:

$$
Y=f\left(h\left(X_{1}, \ldots, X_{n}\right), g\left(W_{1}, \ldots, W_{n}\right)\right)
$$

where $h\left(X_{1}, \ldots, X_{n}\right)$ explains changes in the mean of $Y$ and $g\left(W_{1}\right.$, $\left.\ldots, W_{n}\right)$ explains changes in the precision of $Y$. The explanatory variables represented by $X$ and $W$ can and often are the same, exploring how a particular variable may impact change in both mean and precision of the dependent variable. In this manner, beta regression can be employed in a similar manner to other GLMs by providing estimates of mean change in the dependent variable, but improves upon standard GLMs by modeling possible non-constant changes in variance in the dependent variable.

An interesting challenge to using beta regression is that observations of the dependent variable must fall within the $(0,1)$ interval. This restriction dictates the linking function of the mean
$h\left(X_{1}, \ldots, X_{n}\right)$ must also conform to the $(0,1)$ interval. The 'logit' function is most often selected as the mean link function, providing log-odd mean explanatory regression coefficients that can be easily interpreted after exponentiation as odds ratios (OR) [19] (e.g. a unit change in any $X$ explains a difference in the log-odds of observing $Y$ accounting for error and other $X$ variables in the model). The precision parameter is strictly non-negative; concordantly, a strictly non-negative link function such as the 'natural log' function is used to associate $g\left(W_{1}, \ldots, W_{n}\right)$ with changes in the precision of the dependent variable. Specific inference for precision coefficients is defined as a unit change in any $W$ explains a logarithmic unit change in the precision of $Y$ accounting for error and other $W$ variables in the model. General inference for precision coefficients can be related to changes in variance. Any negative parameter estimate indicates a decrease in precision and subsequent increase in variance; conversely, any positive parameter estimate indicates an increase in precision and subsequent decrease in variance.

The $(0,1)$ interval restriction on the dependent variable makes beta regression naturally adept for modeling percentages. Beta regression has been shown to perform better than linear regression on a transformed variable for percentage data [18]. Any variable that has values outside of the $(0,1)$ range naturally can be scaled as a percentage provided that the variable has a theoretical maximumbyletting $Z=\left[Y^{\prime}(N-1)+0.5\right] / N$ where $Y^{\prime}=[Y-\operatorname{minimum}(Y)] /$ $[\operatorname{maximum}(Y)-\operatorname{minimum}(Y)], N$ is the number of total observations and $Y$ is the original scale measurement [19]. There are distinct advantages to this percentage transformation, as it maintains the shape of a particular variable's distribution allowing non-normal distributions to be modeled easily. However, this transformation shifts any observation exactly equal to 0 or 1 slightly away from 0 or 1 . The relationship between the other values is maintained, but the absolute nature of the value does change. 
Table 1. Univariable OR with $95 \% \mathrm{CI}$, mean and precision $\mathrm{p}$ values associated with 3 -month lesion volumes in the NINDS rt-PA clinical trials

\begin{tabular}{|c|c|c|c|}
\hline Variable & OR (95\% CI) & Mean & Precision \\
\hline \multicolumn{4}{|l|}{ Demographics } \\
\hline Male & $0.946(0.814-1.099)$ & 0.464 & - \\
\hline $\operatorname{Age}^{*}$ & $1.019(1.009-1.028)$ & $<0.001$ & 0.037 \\
\hline \multicolumn{4}{|l|}{ Race } \\
\hline African-American & $0.978(0.594-1.611)$ & 0.930 & - \\
\hline Non-Hispanic Caucasian & $1.108(0.681-1.803)$ & 0.680 & - \\
\hline Hispanic & $1.170(0.665-2.056)$ & 0.586 & - \\
\hline \multicolumn{4}{|l|}{ Comorbidity/risk factor history } \\
\hline Atherosclerosis* & $1.145(0.965-1.359)$ & 0.120 & - \\
\hline Atrial fibrilation* & $1.553(1.189-2.028)$ & 0.001 & 0.174 \\
\hline Other cardiac disease ${ }^{*}$ & $1.132(0.976-1.313)$ & 0.101 & - \\
\hline Prior stroke & $0.987(0.793-1.228)$ & 0.907 & - \\
\hline Aspirin usage & $0.974(0.833-1.138)$ & 0.736 & - \\
\hline Hepatic disease & $0.777(0.495-1.218)$ & 0.271 & - \\
\hline Diabetes* & $1.290(0.972-1.713)$ & 0.078 & 0.018 \\
\hline Hypertension & $1.025(0.876-1.199)$ & 0.761 & - \\
\hline Smoking* & $0.866(0.740-1.014)$ & 0.074 & - \\
\hline Self-reported alcohol usage* & $0.744(0.595-0.930)$ & 0.009 & 0.026 \\
\hline Excessive alcohol by CAGE & $0.862(0.662-1.122)$ & 0.270 & - \\
\hline Consumed alcohol in previous $24 \mathrm{~h}^{*}$ & $0.867(0.707-1.063)$ & 0.169 & - \\
\hline \multicolumn{4}{|l|}{ Clinical features } \\
\hline \multicolumn{4}{|l|}{ Baseline NIHSS* } \\
\hline Weight* & $1.131(1.113-1.150)$ & $<0.001$ & $<0.001$ \\
\hline Early CT findings*, a & $0.996(0.992-1.001)$ & 0.110 & - \\
\hline Early CT findings*,b & $1.820(1.458-2.271)$ & $<0.001$ & 0.088 \\
\hline Admission mean arterial blood pressure ${ }^{*}$ & $2.203(1.683-2.884)$ & $<0.001$ & 0.002 \\
\hline Admission systolic blood pressure & $0.992(0.986-0.999)$ & 0.030 & 0.051 \\
\hline Admission diastolic blood pressure & $0.994(0.990-0.999)$ & 0.010 & 0.019 \\
\hline Admission pulse blood pressure ${ }^{*}$ & $0.998(0.994-1.003)$ & 0.497 & - \\
\hline Admission temperature ${ }^{*}$ & $0.995(0.990-1.000)$ & 0.045 & 0.060 \\
\hline Stroke subtype $(\text { TOAST) })^{*, c}$ & $0.923(0.861-0.991)$ & 0.026 & - \\
\hline Cardioembolic & $6.448(4.597-9.045)$ & $<0.001$ & $<0.001$ \\
\hline Large vessel & $4.567(3.247-6.422)$ & $<0.001$ & $<0.001$ \\
\hline Other & $2.734(1.409-5.302)$ & 0.003 & 0.067 \\
\hline \multicolumn{4}{|l|}{ Trial parameters } \\
\hline rt-PA* & $0.889(0.767-1.031)$ & 0.121 & - \\
\hline Correct dose of rt-PA given $(\%)^{*}$ & $1.004(0.998-1.011)$ & 0.177 & - \\
\hline Time from stroke onset to treatment & $0.999(0.997-1.001)$ & 0.317 & - \\
\hline Time to treatment stratum & $0.972(0.838-1.127)$ & 0.704 & - \\
\hline Trial part 2 & $1.089(0.939-1.264)$ & 0.259 & - \\
\hline \multicolumn{4}{|l|}{ Center ${ }^{*} \mathrm{~d}$} \\
\hline Center 2 & $1.060(0.728-1.541)$ & 0.762 & - \\
\hline Center 3 & $0.894(0.620-1.290)$ & 0.549 & - \\
\hline Center 4 & $1.495(0.934-2.393)$ & 0.094 & 0.086 \\
\hline Center 5 & $1.480(0.931-2.351)$ & 0.097 & 0.184 \\
\hline Center 8 & $1.514(0.922-2.487)$ & 0.101 & 0.082 \\
\hline Center 9 & $1.564(0.881-2.777)$ & 0.127 & 0.124 \\
\hline
\end{tabular}

* Variables meeting selection criteria for multivariable model at $\alpha \leq 0.20$. ${ }^{\text {a }}$ Early CT findings without intravascular thrombus. ${ }^{b}$ Early CT findings including intravascular thrombus. ${ }^{c}$ TOAST groups were compared to small vessel stroke subtype. ${ }^{\mathrm{d}}$ One observation from center 6 was merged into the referent group. 


\section{Multi-Variable Model Construction}

Data from both NINDS rt-PA clinical trials were used to assess whether observed demographic, comorbid/risk factor, clinical feature or study variables were associated with ischemic stroke lesion volumes. As stated above, beta regression can only model variables supported within the $(0,1)$ interval. While a transformation can be used if a theoretical minimum and maximum are known, only the theoretical minimum of brain volume is known $\left(0 \mathrm{~cm}^{3}\right)$. Assuming total brain volume distribution previously reported [20] $\left(1,400 \pm 40 \mathrm{~cm}^{3}\right)$, the NINDS lesion volumes were transformed into percentages using $1,400 \mathrm{~cm}^{3}$ as the theoretical maximum. Development of a multi-variable model followed accepted model development strategy [21], with slight adaptation to compensate for the simultaneous mean and precision modeling of beta regression. This strategy explores baseline variables association with infarct volume using univariable beta regression (only one variable in the model) using a liberal significance criterion $(\alpha=0.20)$. Each variable was selected for multi-variable model inclusion if tests of association with respect to lesion volume for both mean and precision were less than or equal to the criterion level. If a significant association for precision was not detected ( $p>0.20$ ), a constant precision model was fit for the variable of interest and a test of association with respect to lesion volume for the mean was examined for inclusion in the multi-variable model. Categorical variables, including race and TOAST criteria, were examined for association using global tests of association.

All independent variables identified during screening plus variables deemed to be of clinical importance were entered into a multi-variable mean and precision model. A one-step, backward selection removed those variables not meeting the nominal inclusion criterion of $\alpha \leq 0.05$, first removing all non-significant precision variables and then removing all non-significant mean variables. Any variable having a significant precision but non-significant mean effect was examined for model removal by comparing the Bayesian information criteria (BIC) [22] between models with and without the variable. BIC is a measure of goodness of fit that penalizes the model based upon the total number of parameters estimated in the model in order to prevent overfitting. Thus, BIC can be assessed before and after a covariate is removed from the model in order to choose the best parameterization possible. As a general rule of thumb, a BIC change of $\geq 5$ gives moderate evidence of a better model; a change of $\geq 10$ gives conclusive evidence [23].

Upon synthesis of a final model including those variables selected as most strongly associated with stroke volume, differential and modifying effects of one selected explanatory variable on another were investigated by adding interactions between explanatory variables to the final model. These interactions were estimated using the entire data set at an increased rejection criterion of $\alpha=0.10$ according to clinical and statistical guidance $[16,21,24]$. Any significant interactions were investigated using linear contrasts based upon the estimation of the beta regression model from the entire data set and not using subsets of data defined by the interaction. Any continuous variables identified in an interaction were analyzed at the estimated average values of the variables' $10 \mathrm{th}, 25 \mathrm{th}, 50 \mathrm{th}, 75 \mathrm{th}$, and 90 th percentiles in a linear contrast.

As a GLM, an underlying assumption of beta regression is that a linear relationship exists between dependent variable and explanatory variable through the link function. Each continuous variable screened for inclusion was inspected for linearity to the dependent variable through visual inspection of scatterplot and fitted prediction of the dependent variable. Once the final model has been constructed, the differences between prediction and observation can be estimated through residual analysis [25]. The linearity assumption can be further assessed by determining if the residuals are zero-centered and follow a normal distribution. Additionally, a formal test for collinearity does not exist in beta regression. Therefore, Spearman's rank correlation was used for all variables selected for inclusion to detect any pairwise correlations between covariates. Moderately correlated variables $(|\rho| \leq$ 0.40 ) were monitored during the backward selection process; any significant parameter changes in variables upon the exit of a related variable were further investigated for collinearity. All analyses were performed using Stata v11.1 (College Station, Tex., USA).

\section{Results}

\section{Multi-Variable Model}

Twenty of thirty-one candidate variables available in the NINDS rt-PA clinical trials were selected based upon the initial univariable screening (table 1). All continuous variables appeared to maintain linearity with the dependent variable upon scatterplot examination (see online suppl. fig.). Two variables, history of atherosclerosis and admission temperature, had several missing observations (83 and 39 missing observations, respectively); these variables were not included in the multi-variable model but were investigated after the final model had been constructed. Three pairs of variables were highly related to each other leading to one variable from each pair being selected for multi-variable inclusion based upon BIC comparison. Admission mean arterial blood pressure, self-reported alcohol usage, and early CT findings, including intravascular thrombus, were each selected over admission systolic blood pressure, self-reported alcohol consumption in the previous $24 \mathrm{~h}$, and early CT findings without thrombus, respectively. Study specific variables rt-PA indicator, center, time to treatment stratum, and an indicator for part 1 or 2 were included in the multi-variable model regardless of significant association with lesion volume in order to provide adjustment for these study parameters.

At the conclusion of the one-step, backward selection process, rt-PA treatment indicator, age, NIHSS, early CT findings with thrombus, TOAST subtype groups, and study-specific variables remained in the model. Pairwise interactions were investigated between these variables excluding the study-specific variables (center, stratum, and part) and the TOAST subtype due to the sparseness 

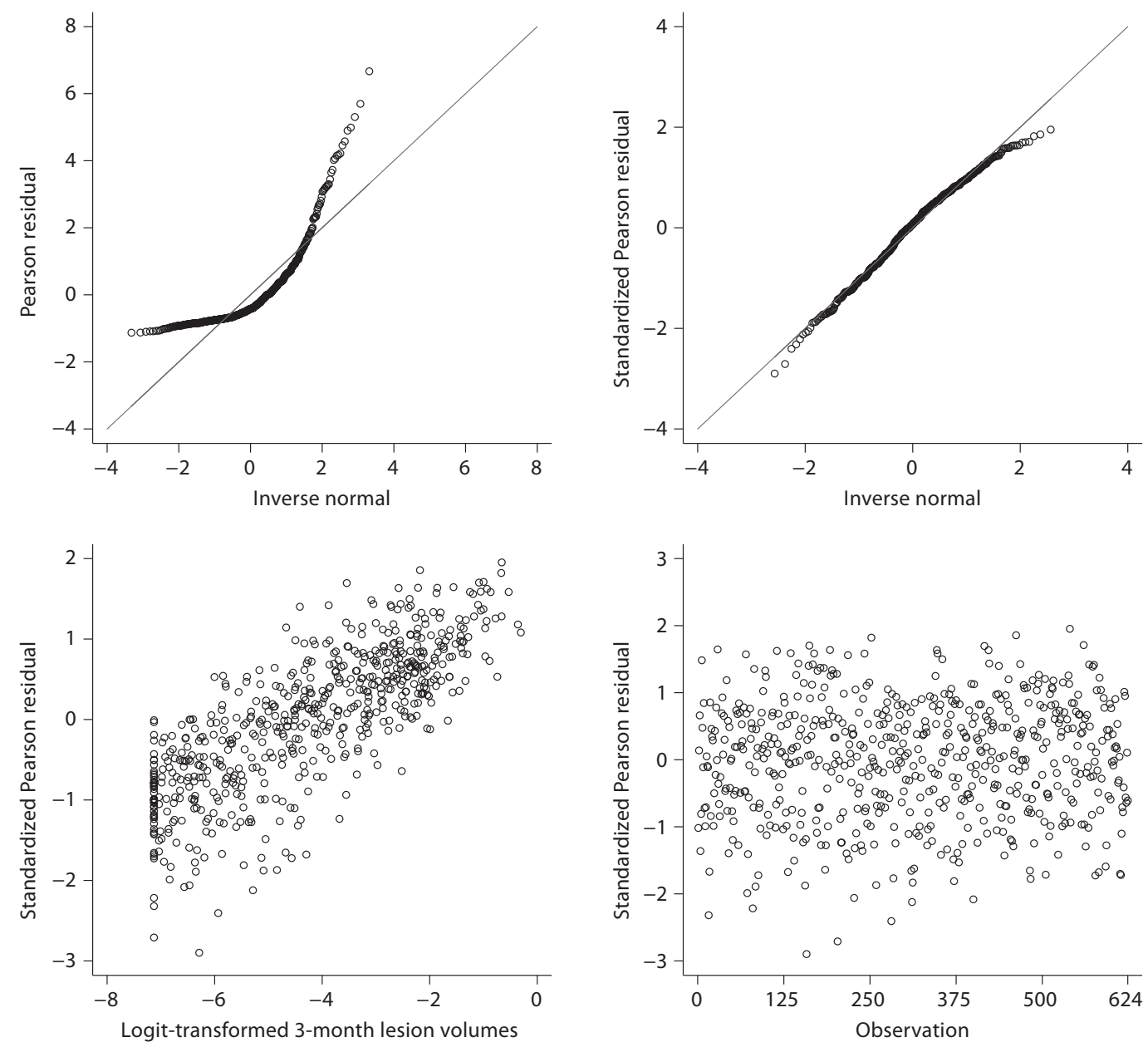

Fig. 2. Residual plots of the final model associating stroke lesion volume $\left(\mathrm{cm}^{3}\right)$ in the NINDS rt-PA clinical trials at 3 months.

of the 'other' category. Three interactions were detected: rt-PA $\times$ age, early CT findings $\times$ NIHSS, and age $\times$ NIHSS, indicating that the association of these variables to lesion volume changed by differing levels of the other variables. Of note, rt-PA indicator, rt-PA $\times$ age interaction, time to treatment and part 1 or 2 indicator were the only variables not remaining in the precision model.

\section{Model Diagnostics}

Examining the residuals confirmed that the final model appropriately fit the data (fig. 2). The Pearson residuals are based upon the differences between the observed and predicted percentages. Since these values are restricted to the $(0,1)$ interval, it is not expected that these residuals would be normal, particularly since by definition the variance changes across the support interval. The Pearson residuals are not centered and clearly not normal as they do not follow the unit normal line. The standardized Pearson residuals are calculated as the difference between observed and predicted data accounting for the transformation used in the estimation of the dependent variable. Accounting for the link function transformations, it is clearly seen that the standardized residuals are zero-centered and follow the unit normal line. Plotting the standardized residuals by the transformed dependent variable further supports the beta regression model assumption that a linear relationship exists between the covariates and the dependent variable via the link functions. Moreover, this 
Table 2. OR and $95 \%$ CI for baseline variable interactions of age $\times$ rt-PA, NIHSS $\times$ early CT finding, age $\times$ NIHSS by percentile* in the NINDS clinical trials

\begin{tabular}{|c|c|c|c|c|c|c|}
\hline & \multicolumn{3}{|c|}{ rt-PA vs. placebo by age interaction } & \multicolumn{3}{|c|}{$\begin{array}{l}\text { Positive vs. negative early CT } \\
\text { finding by NIHSS interaction }\end{array}$} \\
\hline & age, years & $\mathrm{OR}^{\mathrm{a}}$ & $95 \% \mathrm{CI}$ & NIHSS & $\mathrm{OR}^{\mathrm{b}}$ & $95 \% \mathrm{CI}$ \\
\hline 10th percentile & 50.2 & 0.764 & $0.594-0.984$ & 6 & 3.137 & $1.955-5.035$ \\
\hline 25th percentile & 60.0 & 0.864 & $0.731-1.022$ & 9 & 2.749 & $1.875-4.029$ \\
\hline 50th percentile & 68.7 & 0.963 & $0.834-1.111$ & 14 & 2.205 & $1.689-2.879$ \\
\hline 75th percentile & 75.4 & 1.047 & $0.880-1.246$ & 20 & 1.693 & $1.300-2.206$ \\
\hline \multirow[t]{2}{*}{ 90th percentile } & 80.3 & 1.113 & $0.898-1.379$ & 25 & 1.358 & $0.930-1.984$ \\
\hline & \multicolumn{3}{|c|}{ Reference } & & OR & $95 \% \mathrm{CI}$ \\
\hline $\begin{array}{l}\text { Older }(\text { age }=75.4) \\
\text { Mild }(\text { NIHSS }=9)\end{array}$ & \multicolumn{3}{|c|}{$\begin{array}{l}\text { younger }(\text { age }=60.0) \\
\text { mild }(\text { NIHSS }=9)\end{array}$} & & 1.302 & $1.072-1.580$ \\
\hline $\begin{array}{l}\text { Older }(\text { age }=75.4) \\
\text { Severe }(\text { NIHSS }=20)\end{array}$ & \multicolumn{3}{|c|}{$\begin{array}{l}\text { younger }(\text { age }=60.0) \\
\text { mild }(\text { NIHSS }=9)\end{array}$} & & 4.178 & $3.332-5.240$ \\
\hline $\begin{array}{l}\text { Older }(\text { age }=75.4) \\
\text { Mild (NIHSS }=9)\end{array}$ & \multicolumn{3}{|c|}{$\begin{array}{l}\text { younger }(\text { age }=60.0) \\
\text { severe }(\text { NIHSS }=20)\end{array}$} & & 0.342 & $0.261-0.446$ \\
\hline $\begin{array}{l}\text { Older }(\text { age }=75.4) \\
\text { Severe }(\text { NIHSS }=20)\end{array}$ & \multicolumn{3}{|c|}{$\begin{array}{l}\text { younger }(\text { age }=60.0) \\
\text { severe }(\text { NIHSS }=20)\end{array}$} & & 1.096 & $0.923-1.303$ \\
\hline
\end{tabular}

* Percentiles for the continuous variables within the interaction are given. ${ }^{\text {a }}$ OR for rt-PA vs. placebo by each age percentile. ${ }^{\mathrm{b}}$ OR for positive vs. negative CT finding by each NIHSS percentile.

linear relationship is supported by the data without any particular outlier observations observed as exerting undue influence on the model estimation. Finally, plotting the standardized residuals by the observation number sorted by participant entry into the trials indicates a constant variance is maintained across observations as the residuals appear to be randomly scattered within the participant sample and are not heteroskedastic.

\section{Parameter Inference of Mean Model}

Estimates of explanatory variables association with changes in lesion volume are reported as log-odd estimates; OR can be obtained by exponentiation of these estimates, yielding the strength of association between a unit change in an explanatory variable and the odds of having a larger infarct volume. OR and $95 \%$ confidence intervals $(\mathrm{CI})$ for rt-PA $\times$ age, early CT findings $\times$ NIHSS, and age $\times$ NIHSS interactions, while adjusting for all other explanatory variables, were estimated using linear contrasts after fitting the final model by examining each interaction individually and holding all of the other parameters constant (table 2). OR were estimated for each interaction using linear contrasts comparing the 10th, 25th, 50th, 75th, and 90th percentiles of the continuous variables and the indicator variables. In this manner, the difference in lesion volume between treatment and placebo groups can be compared for specific reference ages. Likewise, the difference in lesion volume between those participants with and without early CT finding can be compared at specific NIHSS baseline scores.

Study participants on rt-PA in the 10th age percentile of 50.2 years have significantly decreased odds (table 2 ; $\mathrm{OR}=0.76,95 \%$ CI $0.59-0.98$ ) of having larger 3-month lesion volumes when compared to participants on placebo in the 10th age percentile. However, this is the only statistically significant treatment effect detected for the specific reference ages selected. OR indicating smaller infarct volumes in the treatment group as compared to the placebo group estimated at the 25th and 50th age percentiles are not statistically significant. OR indicating smaller infarct volumes in the placebo group as compared to treatment estimated at the 75th and 90th age percentiles are also not significant. These findings illustrate the powerful mitigating effect age has on stroke outcomes. 
Table 3. Final model precision parameter estimates with 95\% CI associated with 3-month lesion volumes in the NINDS rt-PA clinical trials

\begin{tabular}{lllr}
\hline \multirow{2}{*}{ Variable } & \multicolumn{2}{l}{ Precision model } & \multirow{2}{*}{ p value } \\
\cline { 2 - 3 } & $\begin{array}{l}\text { parameter } \\
\text { estimate }\end{array}$ & $95 \% \mathrm{CI}$ & \\
\hline rt-PA & - & - & \\
Age, years & -0.057 & $-0.089,-0.024$ & 0.001 \\
Baseline NIHSS & -0.297 & $-0.423,-0.171$ & $<0.001$ \\
Early CT findings* & -1.284 & $-2.205,-0.364$ & 0.006 \\
Early CT finding by NIHSS* & 0.045 & $-0.005,0.094$ & 0.077 \\
rt-PA by age & - & - & \\
Age by NIHSS & 0.003 & $0.001,0.005$ & 0.002 \\
TOAST & & & \\
$\quad$ Cardioembolic* & -0.936 & $-1.429,-0.444$ & $<0.001$ \\
$\quad$ Large vessel $^{\text {a }}$ & -1.005 & $-1.485,-0.526$ & $<0.001$ \\
$\quad$ Other & -0.182 & $-1.140,0.776$ & 0.710 \\
Intercept $^{\mathrm{b}}$ & 9.451 & $7.279,11.623$ & $<0.001$ \\
\hline
\end{tabular}

* Early CT findings without intravascular thrombus. ${ }^{\text {a }}$ TOAST groups were compared to small vessel stroke subtype. ${ }^{\mathrm{b}}$ Center, time to treatment, and part indicator were not significant but remained in the final model.
Study participants with early CT findings and a 10th percentile NIHSS of 6 had significantly greater odds (table 2 ; $\mathrm{OR}=3.31,95 \% \mathrm{CI} 1.96-5.04)$ of having a larger infarct volume than those participants in the same NIHSS percentile without early CT findings. A statistically significant increase in odds of having a larger stroke volume is seen for those study participants with early CT findings compared to those participants without early CT findings at every NIHSS percentile except the 90th percentile. Extremely high values of baseline NIHSS reduce the impact of an early CT finding.

Examining the interaction of the two continuous variables, age $\times$ NIHSS, was accomplished in a similar fashion by comparing the 25 th and 75 th percentiles of each variable (table 2). These percentiles defined older participants as 75.4 years of age ( 75 th) and younger participants as 60.0 years of age (25th); mild stroke severity was defined as NIHSS $=9$ (25th) and severe stroke severity as NIHSS $=20$ (75th). Given these reference percentiles, four specific comparisons combining the interaction were made. Participants who were older/mild had 1.30 the odds (95\% CI 1.07-1.58) of having a larger infarct volume than younger/mild participants, while older/mild participants had 0.34 the odds (95\% CI 0.26-0.45) of having a larger infarct volume than the younger/severe participants. Older/severe participants had 4.19 the odds $(95 \%$ CI 3.34-5.25) of having a larger infarct volume than participants who were younger/mild, while older/severe participants had 1.10 the odds (95\% CI $0.92-1.31$ ) of having a larger infarct volume than the young/severe participants. Based upon these results, severe baseline NIHSS is strongly associated with larger lesion volumes, an effect magnified when combined with older age.

\section{Parameter Inference of Precision Model}

Several parameters in the final model were observed to be significantly associated with changes in precision (table 3). General inference is given as changes in the dependent variable's overall variance due to a unit increase in a given explanatory variable. If precision parameter estimates are statistically different from zero, changes in the precision and subsequently variance of the dependent variable are inferred based upon a unit change in the explanatory variable. Considering again the interactions present in the precision model, inference must include both main effects and the interaction term. Thus, examining age and the age $\times$ NIHSS estimates, assuming NIHSS is constant, the overall effect of a unit increase in age is to decrease precision. Subsequently, a decrease in precision leads to an increase in variance as precision resides in the denominator of the variance formula for the beta distribution. Unit increases in NIHSS, assuming other variables are constant, also leads to a decrease in precision and an increase in overall variance of the dependent variable. Early CT findings as well as cardioembolic or large-vessel classified strokes are all associated with decreases in precision and increases in variance. 


\section{Discussion}

Beta regression confirmed the original GEE analysis of the NINDS investigators [16] as the age $\times$ rt-PA interaction, TOAST subtype, and early CT findings $\times$ NIHSS interaction were reported as being statistically significant explanatory variables of 3-month lesion volume. While no specific model estimation was presented, the NINDS investigators described a significant beneficial treatment effect on 3-month lesion volumes for younger participants; younger participants randomized to receive rt-PA had significantly smaller lesion volumes at 3 months than did participants randomized to receive placebo. This treatment effect, as estimated in the GEE analysis, is mitigated as age increases in which older rt-PA recipients have statistically insignificant larger 3-month lesion volumes than placebo recipients.

The beta regression analysis confirms the GEE finding of the rt-PA $\times$ age interaction, although it improves upon the model as beta regression quantifies the treatment effect in terms of an OR. Moreover, beta regression improves on the GEE analysis by observing a previously undetected interaction between age and NIHSS in association with lesion volume. This age $\times$ NIHSS interaction was reported in a previous NINDS subgroup analysis modeling a combined construct of clinical scales [26].

It is equally important to recognize the limitations of this rt-PA analysis on lesion volume. The beta regression analysis of the rt-PA lesion volumes can be considered a realization of the pre-planned secondary analysis. However, this analysis was conducted without consultation or advisement of the NINDS rt-PA study group and should be considered a post hoc analysis. The clinical implications of the age $\times$ rt-PA interaction are difficult to understand. More importantly, the NINDS rt-PA clinical trials were powered to detect clinical differences at $24 \mathrm{~h}$ (part 1) and at 3 months (part 2), not to detect if rt-PA has a differential effect on lesion volume according to age. However, concern regarding the power of beta regression to detect interactions or the possible spurious nature of the findings in general should be tempered by the current analysis' confirmation of the previous GEE analysis.

Beta regression was shown to be a useful mechanism to model the extremely skewed distribution of ischemic stroke infarct volumes. Beta regression synthesized a final model using the NINDS rt-PA clinical trials data that was similar to the previously reported model, but was able to improve upon the previous analysis by modeling the data more completely, particularly in associating explanatory variables with changes in both the mean and the precision of the dependent variable. This improved modeling led to the finding of the additional interaction of age $\times$ NIHSS explanatory variables associated with infarct volumes.

It was unclear at the outset how beta regression would compare to the original GEE cube-root transformation analysis of the rt-PA trials. The similarity of the results from the two analytical models indicates that beta regression is a legitimate alternative to the previous analytical method. Moreover, the estimation of changes in precision by beta regression supports clinical intuition that more variability in lesion volume occurs with increases in age, NIHSS, and prior stroke findings. However, beta regression provides two distinct advantages over the previous model. It inherently models percentages which allowed a more simple and intuitive transformation of lesion volumes than previously achieved. More importantly, it offers inference in terms of OR, allowing for an interpretation of treatment effect that was previously unavailable.

\section{Acknowledgments}

Dr. Swearingen received support from NINDS T32 NS4800701A1. Dr. Tilley received support from NINDS U01NS043127. Dr. Bandyopadhyay received research support from National Center for Research Resources (NCRR) P20 RR017696-06. The authors extend special thanks to the reviewers in critically examining the manuscript.

\section{Disclosure Statement}

The authors have no conflicts of interest or disclosures to report.

References

1 Donnan GA, Fisher M, Macleod M, Davis SM: Stroke. Lancet 2008;371:1612-1623.

$\checkmark 2$ van Everdingen KJ, van der Grond J, Kappelle LJ, Ramos LMP, Mali WPTM: Diffusion-weighted magnetic resonance imaging in acute stroke. Stroke 1998;29:17831790.

-3 Schiemanck SK, Kwakkel G, Post MWM, Prevo AJH: Predictive value of ischemic lesion volume assessed with magnetic resonance imaging for neurological deficits and functional outcome post stroke: a critical review of the literature. Neurorehabil Neural Repair 2006;20:492-502.

4 González RG: Imaging-guided acute ischemic stroke therapy: from 'time is brain' to 'physiology is brain'. Am J Neuroradiol 2006; 27:728-735. 
5 Saunders DE, Clifton AG, Brown MM: Measurement of infarct size using MRI predicts prognosis in middle cerebral artery infarction. Stroke 1995;26:2272-2276.

66 Thijs VN, Lansberg MG, Beaulieu C, Marks MP, Moseley ME, Albers GW: Is early ischemic lesion volume on diffusion-weighted imaging an independent predictor of stroke outcome? A multivariable analysis. Stroke 2000;31:2597-2602.

7 Wardlaw JM, Keir SL, Bastin ME, Armitage PA, Rana AK: Is diffusion imaging appearance an independent predictor of outcome after ischemic stroke? Neurology 2002;59: 1381-1387.

$\checkmark 8$ MR Stroke Collaborative Group: Proof-ofprinciple phase II MRI studies in stroke: sample size estimates from dichotomous and continuous data. Stroke 2006;37:2521-2525.

>9 Tong DC, Yenari MA, Albers GW, O’Brien M, Marks MP, Moseley ME: Correlation of perfusion- and diffusion-weighted MRI with NIHSS score in acute ( $<6.5$ hour) ischemic stroke. Neurology 1998;50:864-870.

$\checkmark 10$ Beaulieu C, de Crespigny A, Tong DC, Moseley ME, Albers GW, Marks MP: Longitudinal magnetic resonance imaging study of perfusion and diffusion in stroke: evolution of lesion volume and correlation with clinical outcome. Ann Neurol 1999;46:568-578.
11 Warach S, Pettigrew LC, Dashe JF, Pullicino P, Lefkowitz DM, Sabounjian L, Harnett K, Schwiderski U, Gammans R: Effect of citicoline on ischemic lesions as measured by diffusion-weighted magnetic resonance imaging. Ann Neurol 2000;48:713-722.

12 Engelter ST, Wetzel SG, Radue EW, Rausch M, Stech AJ, Lyrer PA: The clinical significance of diffusion-weighted MR imaging in infratentorial strokes. Neurology 2004;62: 574-580.

13 Lu M, Chase G, Li S: Permutation tests and other test statistics for ill-behaved data: experience of the NINDS t-PA stroke trial. Commun Stat Theory Methods 2001;30: 1481-1496.

$>14$ NINDS rt-PA Stroke Study Group: Tissue plasminogen activator for acute ischemic stroke. N Engl J Med 1995;333:1581-1587.

15 Brott T, Adams HP, Olinger CP, Marler JR, Barsan WG, Biller J, Spilker J, Holleran R, Eberle R, Hertzberg V: Measurements of acute cerebral infarction: a clinical examination scale. Stroke 1989;20:864-870.

16 NINDS rt-PA Stroke Study Group: Effect of intravenous recombinant tissue plasminogen activator on ischemic stroke lesion size measured by computed tomography. Stroke 2000;31:2912-2919.

17 Adams HP Jr, Bendixen BH, Kappelle LJ, Biller J, Love BB, Gordon DL, Marsh EE 3rd: Classification of subtype of acute ischemic stroke: definitions for use in a multicenter clinical trial. Stroke 1993;24:35-41.

18 Paolino P: Maximum likelihood estimation of models with beta-distributed dependent variables. Polit Anal 2001;9:325-346.
19 Smithson M, Verkuilen J: A better lemon squeezer? Maximum-likelihood regression with beta-distributed dependent variables. Psychol Methods 2006;11:54-71.

20 Lyden PD, Zweier R, Mahdavi Z, Lonzo L: A rapid, reliable, and valid method for measuring infarct and brain compartment volumes from computed tomographic scans. Stroke 1994;25:2421-2428.

21 Hosmer DW, Lemeshow S: Model-Building Strategies and Methods for Logistic Regression: Applied Logistic Regression, ed 2. New York, John Wiley and Sons, 2000.

$\checkmark 22$ Schwarz GE: Estimating the dimension of a model. Ann Stat 1978;6:461-464.

23 Raftery AE: Bayesian model selection in social research. Sociol Methodol 1995;25:111163.

24 Altman DG, Schulz KF, Moher D, Egger M, Davidoff F, Elbourne D, Gøtzsche PC, Lang $\mathrm{T}$, for the CONSORT Group: The revised CONSORT statement for reporting randomized trials: explanation and elaboration. Ann Intern Med 2001;134:663-694.

25 Rocha AV, Simas AB: Influence diagnostics in a general class of beta regression models. Test 2011;20:95-119.

26 NINDS rt-PA Stroke Study Group: Generalized efficacy of t-PA for acute stroke: subgroup analysis of the NINDS t-PA stroke trial. Stroke 1997;28:2119-2125. 